\title{
Connaissances Attitudes et Pratiques des agents de santé de la ville de Ségou sur les cancers de l'enfant
}

\section{Knowledge Attitudes and Practices of Health Workers in the City of Ségou on Childhood Cancers}

Bah $A^{1}$, Bagayoko $\mathrm{TB}^{2}$, Kassogué $\mathrm{A}^{1}$, Koné $\mathrm{SI}^{4}$, Samaké $\mathrm{B}^{3}$, Traoré $\mathrm{T}^{5}$, Dramé $\mathrm{MB}^{6}$, Harber $\mathrm{B}^{1}$, Guindo $\mathrm{M}^{1}$, Thiéro $\mathrm{A}^{1}$, Diall $\mathrm{M}^{7}$, Keita $\mathrm{M}^{3}$, Coulibaly $\mathrm{DS}^{9}$, Coulibaly $\mathrm{A}^{8}$, Togo $\mathrm{MA}^{8}$, Fofana $\mathrm{A}^{10}$, Sanogo $\mathrm{A}^{11}$, Diakité $\mathrm{L}^{12}$

\section{DOI : 10.53318/msp.v11i1.1896}

1 : Service de Pédiatrie/ de l'Hôpital Nianankoro Fomba de Ségou, Mali (HNF, Ségou, Mali)

2. Service de médecine légale/travail, HNF, Ségou, Mali

3. Service de chirurgie générale, HNF, Ségou, Mali

4. Service d'urologie, HNF, Ségou, Mali

5. Service de gynécologie et d'obstétrique, HNF, Ségou, Mali

6. Service de chirurgie maxillo faciale, HNF, Ségou, Mali

7. Unité du Système d'Information Hospitalière, HNF, Ségou, Mali

8. Service d'ophtalmologie, HNF, Ségou, Mali

9. Service de médecine générale, HNF, Ségou, Mali

10. Service d'otorhinolaryngologie, HNF, Ségou, Mal

11. Service de cardiologie, HNF, Ségou, Mali

12. Centre de santé de référence Famory Doumbia, Ségou, Mali

*Correspondant : Dr Adama Bah, Spécialiste en Pédiatrie, chef de service de Pédiatrie de l'Hôpital Nianankoro Fomba de Ségou (Mali), Tel : 002237616 5941, Email : bah750@gmail.com

Conflit d'intérêt : aucun

\section{Résumé :}

Introduction : Les cancers de l'enfant sont rares et représentent environ $1 \%$ de la globalité des cancers. Cependant, leur sévérité, la durée de leur traitement et leur évolution au long cours en font un problème de santé publique majeur. Le but de notre étude était d'évaluer les connaissances, attitudes et pratiques des agents de santé de la ville de Ségou sur les cancers de l'enfant. Méthodologie : II s'agissait d'une étude transversale, descriptive et analytique menée auprès des agents de santé de la ville de Ségou. Résultats : Trois cent dix agents de santé de la ville de Ségou ont répondu à notre questionnaire dont 32 médecins généralistes, 31 médecins spécialistes, 32 assistants médicaux, 41 infirmiers du second cycle, 88 infirmiers de premier cycle, 44 aides-soignants et Matrones, 13 techniciens de laboratoire 29 sages-femmes Les agents de santé qui ont déclaré que les cancers de l'enfant sont des affections malignes représentaient $55,16 \%$. Dans notre étude, $73,5 \%$ des agents de santé déclaraient n'avoir pas suspecté ou diagnostiqué un cancer de l'enfant durant leur carrière, $75 \%$ n'ont jamais participé à la prise en charge d'un enfant atteint d'un cancer au cours de leur carrière. Conclusion : Le domaine de l'oncologie pédiatrique est méconnu des agents de santé de la ville de Ségou, en tant que 1ers contacts lors de l'apparition de symptômes pouvant faire évoquer un cancer. La discipline est peu enseignée dans le cursus de formation des agents de santé au Mali.

Mots clés : cancers-enfant-agents de santé de Ségou

\section{Abstract:}

Introduction: childhood cancers are rare and account for about $1 \%$ of all cancers. However, their severity, duration of treatment and long-term progression make them a major public health problem. The purpose of our study was to evaluate the Attitudes and Practices knowledge of health workers in the city of Ségou on childhood cancers. Methodology: this is a cross-sectional descriptive epidemiological study of health workers in the city of Ségou. Result: three hundred and ten health workers from the city of Ségou responded to our questionnaire, including 32 general practitioners, 31 specialist doctors, 32 medical assistants, 41 second-cycle nurses, 88 undergraduate nurses 44 nursing assistants and Matrons 13 laboratory technicians 29 midwives Health workers who report child cancer as a malignant disease accounted for $55.16 \%$. In our study, $73.5 \%$ of health workers report not having suspected or diagnosed childhood cancer during their career; $75 \%$ have never participated in the care of a child with cancer during their career. Conclusion: the field of pediatric oncology is unknown to health workers in the city of Ségou, as the first contacts when symptoms may suggest cancer. The discipline is not taught much in the training curriculum of health workers in Mali.

Keywords: cancers- child- Ségou health workers

\section{I-Introduction}

Les cancers de l'enfant sont rares et représentent environ $1 \%$ de la globalité des cancers [1]. Cependant, leur sévérité, la durée de leur traitement et leur évolution au long cours en font un problème de santé publique majeur. Dans les pays industrialisés, les cancers représentent la deuxième cause de mortalité des enfants entre 1 et 14 ans après les accidents. On estime aujourd'hui qu'un adulte sur 500 est un survivant d'une maladie cancéreuse survenue dans l'enfance [2]. Chaque année en Europe, un enfant sur 7000 âgé de 0 à 14 ans, développera un cancer, ce qui représente 13000 nouveaux cas de cancers pédiatriques diagnostiqués annuellement [2].

Les progrès réalisés au cours des dernières décennies dans les taux de guérison des cancers de l'enfant ne sont pas associés à la précocité du diagnostic mais au progrès de la prise en charge thérapeutique [3]. Cette prise en charge est pluridisciplinaire et nécessite l'implication d'équipes spécialisées [4].

Si le taux de survie à cinq ans d'un enfant atteint de cancer s'est progressivement amélioré dans les pays développés grâce aux progrès diagnostiques et thérapeutiques, passant de $56 \%$ en 1974 à plus de $81 \%$ en 2000 aux Etats-Unis, il n'en est pas de même en Afrique subsaharienne où le diagnostic de cancer est jusqu'à récemment synonyme d'une sentence de mort programmée [5]. 
Au Mali, en 2010, l'incidence annuelle moyenne des cancers était de 115 nouveaux cas, qui sont au-dessous de la réalité, vue : la pauvreté, la méconnaissance des premiers signes par les agents de santé et le recours à la médecine traditionnelle et en absence de registre fiable Ceci fait que beaucoup d'enfants maliens atteints de cancer ne sont pas diagnostiqués [6]. Les signes d'appel sont le plus souvent banals et peuvent passer longtemps inaperçus, souvent d'apparition et d'évolution rapide alors que l'enfant conserve un bon état général [7]. II semble donc nécessaire d'évaluer les connaissances des agents de santé dans le domaine de l'oncologie pédiatrique puisqu'ils sont le plus souvent les premiers à être consultés en cas d'apparition des premiers symptômes. L'objectif était d'évaluer les connaissances et attitudes des agents de santé de la ville de Ségou sur les cancers de l'enfant.

\section{III-Matériel et Méthodes}

1-Cadre d'étude : II s'agissait d'une étude transversale multicentrique, descriptive et analytique qui a été réalisée dans les structures de santé (publiques et privées) de la ville de Ségou. Qui sont des établissements de première et de deuxième référence.

2-Type et Période de l'étude :

II s'agissait d'une étude transversale multicentrique, descriptive et analytique, qui s'est déroulée du 1er juin au 31 juillet 2019.

\section{3.Échantillonnage :}

Pour calculer la taille de notre échantillon, nous avons considéré le nombre total de personnel de santé dans la ville de Ségou dénombré dans l'annuaire statistique de 2016 comme la taille de notre population cible $(n=531)$. Nous avons calculé la taille de l'échantillon avec la formule de Daniel Schwartz:

$$
n=t^{2} \times p \times(1-p) / m^{2}
$$

- $\quad \mathrm{n}$ : Taille d'échantillon minimale pour l'obtention de résultats significatifs pour un événement et un niveau de risque fixé

- $\quad t$ : test de l'écart réduit $=1,96$ pour un risque $a$ de 0.05

- $\quad p$ : proportion estimée de la population 0,075

- $m$ : Précision voulue $(0,05)$

$n=1,96^{2} \times 0,075 \times 0,925 / 0,05^{2}=106,6$ soit 107 personnes

4-Population de l'étude : La population cible étudiée était constituée par le personnel soignant qualifié et non qualifié impliqué dans les soins des structures ayant servi de cadre pour notre étude.

5-Critères de l'étude

a-Critères d'inclusion :

- Les structures : il s'agissait des centres de santé publics et privés administrant des soins médicaux. Nous avons sélectionné les 6 centres de santé communautaire, le centre de santé de référence Famory Doumbia, l'hôpital régional et trois cliniques médicales en raison de leur représentativité en termes de recrutement et de leur rôle reconnu dans la prise en charge des pathologies médicales de l'enfant.

-Le personnel enquêté : nous avons retenu uniquement les personnels soignants (médecins infirmières sagesfemmes ; assistants médicaux, aides-soignants et matrones) en service dans les structures concernées.

b-Critères de non-inclusion : N'ont pas été inclus dans l'étude : le personnel des centres de santé qui ne répondait pas aux critères de prise en charge des maladies de l'enfant, le personnel retissant et le personnel non disponible pendant la période de l'étude.

6-Méthode de collecte des données :

Elles ont été collectées sur un questionnaire préalablement établi. Le questionnaire a été administré directement, en tête à tête au niveau des centres ciblés au personnel inclus dans l'étude par l'enquêteur. II s'agissait d'un questionnaire avec des réponses ouvertes et fermées administrées directement.

Paramètres à étudier :

-Données générales sur le personnel enquêté : Sexe, structure d'exercice, grade professionnel, nombre d'années d'expérience dans la santé, formation initiale sur les cancers de l'enfant,

-Données générales sur les connaissances, les attitudes et les pratiques sur les cancers de l'enfant

\section{7-Analyse des données}

Les données ont été saisies et analysées par le logiciel SPSS IBM version 12. Nous avons présenté nos variables quantitatives sous forme de moyenne ou de médiane avec un minimum et un maximum. Les variables qualitatives ont été décrites sous forme de pourcentage. Les tests Chi2 ont été utilisés et il existait une relation statistiquement significative si $p<0,05$

\section{IV-Résultats :}

Effectif global :

Durant notre période d'étude, trois cents dix agents de santé ont répondu à notre questionnaire soit un taux de participation de $79 \%$. On notait $20,3 \%$ de médecins ; le secteur public représentait $94 \%$ et $74 \%$ des enquêtés étaient des salariés. L'expérience professionnelle était de 1 à 5 ans dans $42 \%$ des cas. L'âge moyen des enquêtés était de 35 ans avec extrêmes allant de 18 à 59 ans. La moyenne d'âge d'exercice professionnelle était de 8,7 ans (minimum 1 an et maximum 39 ans). Le sex- ratio était de 0,78 en faveur des femmes (Tableau I)

Connaissances des agents de santé dans le domaine de l'oncologie pédiatrique

Dans notre étude, $55,16 \%$ des enquêtés déclaraient que les cancers de l'enfant sont des affections malignes ; $73,50 \%$ ne connaissaient pas la fréquence des cancers de l'enfant dans le monde. L'âge d'apparition des cancers de l'enfant était méconnu par $50 \%$ des cas parmi eux 50 $\%$ de ces enquêtés travaillaient à l'hôpital régional de Ségou $(p=0,0029)$. L'existence des facteurs de prédisposition aux cancers de l'enfant n'était pas connue par $55 \%$ des participants. L'existence de syndromes de 
prédisposition génétique nécessitant un dépistage dès le jeune âge n'était pas connue par $77 \%$ des participants. Cependant l'existence de signes spécifiques faisant évoqués un cancer chez l'enfant était connue par $72 \%$ des participants ainsi que l'existence de malformations associées à la survenue des cancers de l'enfant était connue par $79 \%$ des enquêtés parmi eux $23 \%$ des médecins $(p=0,04)$.

Les manifestations cliniques des cancers de l'enfant n'étaient connues par $59 \%$ des enquêtés ; $66 \%$ ne connaissent pas les types de cancers les plus fréquents chez l'enfant. Les différentes modalités de traitement des cancers de l'enfant n'étaient connues par $71 \%$ des enquêtés et $44 \%$ déclaraient que les cancers de l'enfant n'étaient pas curables ils étaient des médecins dans 5,48 $\%$ des cas $(p=0,049)$ et exerçaient à l'hôpital de Ségou dans $36 \%$ des cas ' $p=0,0037$ ). (Tableau : II ; III ; IV ; V ;). Expérience des agents de santé dans le domaine de l'oncologie pédiatrique

Dans notre étude, $73,5 \%$ des enquêtés déclaraient n'avoir pas suspecté ou diagnostiqué un cancer de l'enfant durant leur carrière dont $56,58 \%$ étaient des infirmiers ( $p=$ $0,00001) ; 75 \%$ n'avaient jamais référé ou participé à la prise en charge d'un enfant atteint d'un cancer au cours de leur carrière dont $11,21 \%$ étaient des médecins ( $p=$ $0,00001)$ et $43,53 \%$ travaillaient à l'hôpital régional $(p=$ $0,00001) ; 81 \%$ des enquêtés pensaient ne pas être suffisamment informés sur les signes d'appel du cancer de l'enfant ; $92 \%$ n'avaient eu un enfant atteint de cancer dans l'entourage familiale ; $94 \%$ n'étaient pas formés sur les cancers de l'enfant dont 19,24\% étaient des médecins ; $95 \%$ n'avaient jamais travaillé dans un service d'oncologie médicale ou dans une unité d'oncologie pédiatrique dont $19,04 \%$ des médecins et $60 \%$ ne connaissaient pas l'existence d'une unité d'oncologie pédiatrique au Mali dont 28,49\% (figure1) .

Attitude des agents de santé dans le domaine de l'oncologie pédiatrique

Dans notre étude, les agents de santé déclaraient référer les cas suspects de cancers de l'enfant vers l'oncologie pédiatrique dans $66,1 \%$ et vers la pédiatrie générale dans $23,5 \%$. Les enquêtés qui souhaitaient participer à des enseignements post universitaire sur les cancers de l'enfant représentaient 95,2\%.

\section{V-Discussion : \\ Effectif global}

Dans notre étude, les infirmiers (infirmiers $1^{\mathrm{er}} \mathrm{cycles}$, infirmiers d'état sages-femmes et techniciens de laboratoire) étaient les plus représentés avec $55,2 \%$ suivi des médecins avec $20,3 \%$ des cas, les aides-soignants et les matrones avec 14,2\% chacun et enfin les assistants médicaux (10,3\%). Cette forte représentation des infirmiers pourrait être expliquée d'une part par l'existence de plusieurs écoles de formation des agents de santé et d'autre part par l'organisation de la pyramide sanitaire de notre pays.
Trois cents dix ont participé à notre étude soit un taux de participation de $79 \%$. Nos résultats sont supérieurs à ceux de Marly $A$ dans une étude réalisée à Nancy qui a trouvé un taux de participation de 7,5\% [8]. Cette différence pourrait s'expliquée par la composition de notre population d'étude qui était constituée de tous les grades professionnels de la santé alors que la population d'étude de Marley A était constituée de médecins généralistes et de pédiatres.

L'âge moyen des participants était de 35 ans $\pm 65,74$ ans (Extrêmes : 18 ans et 59 ans). II s'agissait de 136 hommes et 174 femmes. Le sexe ratio était de 0,78 en faveur des femmes. Nos résultats sont différents de celui d'Aurélie Marly qui avait trouvé 79 hommes et 73 femmes avec un sexe ratio en faveur des hommes [8]. Cette différence pourrait être liée la différence de grade professionnelle entre les deux populations d'étude.

\section{Expérience professionnelle :}

Les agents de santé ayant participé à notre étude exerçaient la profession depuis en moyen 8,71 ans avec un écart type de 47,29 ans (minimum 1an, maximum 39 ans). Ces résultats sont largement inférieurs à celui d'Aurélie Marly qui avait trouvé une durée moyenne d'exercice de 21,8 avec un écart type de 12,9 ans et des extrêmes de 1 an et 44 ans [8]. Cette différence pourrait s'expliquer par la mobilité des agents de santé qui après leurs titularisations dans la fonction rejoignent la ville de Bamako. En Inde l'expérience professionnelle a été indentifiée comme facteurs [9]. II en était de même dans d'autres études [10-12]

Connaissances des agents de santé de la ville de Ségou dans le domaine d'oncologie pédiatrique :

La moitié des agents de santé avait déclaré que les cancers n'étaient pas des affections malignes, parmi eux certains pensaient que les cancers de l'enfant étaientdes maladies infectieuses $(6,13 \%)$, des maladies héréditaires $(6,13 \%)$, ou des maladies bénignes $(5,80 \%)$. La majorité ne connaît pas les facteurs prédisposant aux cancers de l'enfant et plus du quart ne connaissaient pas les signes spécifiques évoquant un cancer dans cette tranche d'âge. Ces résultats sont inférieurs à ceux d'Aurélie Marly qui avait trouvé que $47 \%$ d'agents de santé ne connaissaient pas l'existence de signes spécifiques faisant évoquer un cancer chez l'enfant [8]. .

Dans notre étude, près d'un quart d'agents de santé déclaraient ne pas connaitre l'existence de malformations associées à la survenue de cancers chez l'enfant et qui nécessitent un dépistage, et près d'un quart ne connaissaient pas l'existence des syndromes de prédisposition génétique. Ces résultats sont inférieurs à ceux d'Aurélie Marly qui avait trouvé que $60 \%$ d'agents de santé ne connaissaient l'existence de malformations associées à la survenue de cancers de l'enfant nécessitant un dépistage [8]. Plus de la moitié des agents de santé de la ville de Ségou n'avaient pas d'idée sur les manifestations cliniques ainsi que sur les types les plus fréquents des cancers de l'enfant et la majorité déclarait 
ne pas connaitre les différentes modalités de traitement des cancers dans cette tranche d'âge.

Près de la moitié des agents de santé avaient mentionné que les cancers de l'enfant ne sont pas curables. La grande majorité ne connaît pas le taux de survie à 10 ans des cancers de l'enfant dans les pays développés. Dans l'étude menée par Aurélie Marly, $36 \%$ pédiatres et médecins ne connaissaient pas le taux de survie à 10 ans des cancers de l'enfant dans les pays développé [8]. Cette différence pourrait s'expliquée par la différence de grade professionnelle entre nos deux populations, notre étude avait concerné toutes les couches socio- professionnelles de la santé alors qu'Aurélie ne s'était intéressée qu'aux médecins.

Expériences des agents de santé dans le domaine de l'oncologie pédiatrique

Dans notre série, la plus part des agents de santé n'avaient jamais suspecté ou diagnostiqué un cancer chez l'enfant. Parmi eux, plus de la moitié étaient des infirmiers (1ére cycle, second cycle et assistants médicaux). Plus de la moitié n'étaient pas au courant de l'existence d'une unité de prise en charge des cancers de l'enfant au Mali, parmi eux, plus d'un quart étaient des médecins. Par ailleurs, la majorité des agents de santé n'avait jamais référé ou participé à la prise en charge d'un enfant atteint de cancer, et ils déclaraient ne pas être suffisamment informés des signes d'appel du cancer chez l'enfant. Ces résultats sont comparables à ceux d'Aurélie Marly qui avait trouvé que $91,6 \%$ des agents de santé pensaient ne pas être suffisamment informés sur les signes d'appel des cancers de l'enfant [8].

Attitudes des agents de santé dans le domaine de l'oncologie pédiatrique

Plus de la moitié des agents de santé enquêtés déclaraient référer vers l'oncologie pédiatrique les cas suspects de cancers de l'enfant et la grande majorité souhaitaient participer à des formations continues sur les cancers de l'enfant. Ces résultats montrent à suffisance le besoin de formation des agents de santé afin de pouvoir poser le diagnostic et orienter les patients vers les structures de prise en charge. La formation de base des agents de santé ainsi que leur formation continue doivent constituer un passage obligatoire dans les pays en développement comme le Mali où une véritable approche du cancer de l'enfant n'est pas encore instituée. Le meilleur pronostic et l'augmentation de l'âge de survie des enfants atteints de cancers sont intimement liés au diagnostic et à l'initiation précoce d'une thérapeutique.

\section{Conclusion :}

Comme le montre notre étude, le domaine de l'oncologie pédiatrique est méconnu des agents de santé de la ville de Ségou, alors qu'ils sont les premiers consultés lors de l'apparition de symptômes pouvant faire évoquer un cancer. En effet, il s'agit d'une discipline peu enseignée à la faculté de médecine et dans les écoles de formation socio-sanitaires. Ainsi, ces agents de santé seraient plutôt favorables à participer à des formations continues sur les cancers de l'enfant qui auront certainement un impact positif sur l'amélioration de la prise en charge et sur le pronostic de ces patients.

\section{Références}

1- Steliarova-Foucher E, Stiller C, Kaatsch $P$, et al. Geographical patterns and time trends of cancer incidence and survival among children and adolescents in Europe since the 1970s (the ACCISproject): an epidemiological study. Lancet 2004;364(9451):2097-105.

2- Henze. Foreword. Eur J Cancer 2006;42:1913-4

3- Marie-Anne WEIDNER. Comment raccourcir le délai diagnostic du cancer chez l'enfant et l'adolescentProposition de moyen de sensibilisation. Thèse de médecine [Faculté de médecine, Nancy 1] : Université de Lorraine; 2015.

4-Lobut J-B, Merbaï S, Asensi D. Prise en charge de proximité des enfants atteints de cancer. Médecine Thérapeutique Pédiatrie. 1 mai 2003;6(3):129-34.

5-Kadan-Lottick NS.Cancer and benign tumors. In: Kliegman Behrman Jenson Stanton eds. Nelson Texbook of Pediatrics 18 thed Philadelphia. Saunders Elsevier 2007: 2097-162.

6- Togo B, Traoré F, Togo A P, et al. Epidémiologie et pronostic des cancers pédiatriques au CHU Gabriel Touré de Bamako (Mali). Médecine et santé Tropical 2014 ; 24 : 68-72.

7- Pr Pascal Chastagner. Cancer de l'enfant- Particularités épidémiologiques, diagnostiques et thérapeutiques. Rev Prat. 2016;66.

8-Aurélie Marly. Evaluation à l'aide d'un questionnaire de la possibilité de créer un réseau d'oncologie pédiatrique CHRU de Nancy. Médecine de ville. Science du vivant [qbio]. 2017 ; hal- 01932094 . Thèse de médecine. Université de Lorraine.

9- Faruqui N, Bernays S, Martiniuk A, Abimbola S, Arora $\mathrm{R}$, Lowe J, et al. Access to care for childhood cancers in India: perspectives of health care providers and the implications for universal health coverage. BMC Public Health. 3 nov 2020;20:1641.

10-Nahar VK, Hasani Z, Martin B, Boyas JF, Chabok R, Philip LS, et al. Perceptions and Practices of the Iranian Population regarding Skin Cancers: A Literature Review. J Skin Cancer. 2017;2017:4934108.

11-Khamisy-Farah R, Adawi $M$, Jeries-Ghantous $H$, Bornstein J, Farah R, Bragazzi NL, et al. Knowledge of Human Papillomavirus (HPV), Attitudes and Practices Towards Anti-HPV Vaccination Among Israeli Pediatricians, Gynecologists, and Internal Medicine Doctors: Development and Validation of an $\mathrm{Ad} \mathrm{Hoc}$ Questionnaire. Vaccines. 21 oct 2019;7(4):157.

12- Suh E, Daugherty CK, Wroblewski K, Lee H, Kigin ML, Rasinski KA, et al. General Internists' Preferences and Knowledge About the Care of Adult Survivors of Childhood Cancer. Ann Intern Med. 7 janv 2014;160(1):11-7. 
Liste des tableaux et figures

Tableau I : Répartition des agents de santé selon leurs statuts sociodémographiques et professionnels

\begin{tabular}{lll}
\hline \hline Statuts & $\mathbf{n}$ & $\%$ \\
\hline \hline Grade Professionnel & & \\
Aides-soignants & 33 & 10,6 \\
Assistant médicaux & 32 & 10,3 \\
Infirmier 1er cycle & 88 & 28,4 \\
Infirmier d'Etat & 41 & 13,2 \\
Matrones & 11 & 3,6 \\
Médecin généraliste & 32 & 10,3 \\
Médecin spécialiste & 31 & 10,0 \\
Sage-femme & 29 & 9,4 \\
Technicien de Laboratoire & 13 & 4,2 \\
Sexe & & \\
Masculin & 136 & 44 \\
Féminin & 174 & 56 \\
& & \\
Lieu d'exercice & & \\
Cabinets Privés & 9 & 2,90 \\
Cliniques privées & 11 & 3,50 \\
CSCOM & 98 & 31,60 \\
CSRéf & 30 & 9,70 \\
HNF & 162 & 52,30 \\
Expérience professionnelle & & \\
1-5 ans & 130 & 41,94 \\
6-10 ans & 79 & 25,48 \\
11-15 ans & 65 & 20,97 \\
16-20 ans & 19 & 6,13 \\
21-25 ans & 7 & 2,26 \\
> 30 ans & 3 & 0,97 \\
Total & 7 & 2,26 \\
\hline \hline & 310 & 100 \\
\hline
\end{tabular}

Tableau II : Répartition des agents de santé selon la réponse à la question : Qu'entendez- vous par cancers de l'enfant?

\begin{tabular}{lll}
\hline $\begin{array}{l}\text { Qu'entendez- vous par } \\
\text { cancers de l'enfant }\end{array}$ & $\mathbf{n}$ & $\%$ \\
\hline \hline Affection maligne & 171 & 55,16 \\
\hline Affection bénigne & 18 & 5,80 \\
\hline Maladie infectieuse & 19 & 6,13 \\
\hline Maladie héréditaire & 19 & 6,13 \\
\hline Ne sait pas & 52 & 16,78 \\
\hline Total & 310 & 100 \\
\hline \hline
\end{tabular}

Tableau III : Répartition des agents de santé selon la réponse à la question : Quels sont les facteurs Prédisposant aux cancers de l'enfant?

\begin{tabular}{lll}
\hline $\begin{array}{l}\text { Quels sont les facteurs } \\
\text { Prédisposant } \\
\text { cancers de l'enfant }\end{array}$ & $\mathrm{n}$ & $\%$ \\
\hline \hline Tabagisme & 31 & 10 \\
Stress & 9 & 2,90 \\
Irradiation lonisante & 35 & 11,29 \\
Héréditaire & 35 & 11,29 \\
Produit chimique & 30 & 9,68 \\
Ne sait pas & 170 & 54,84 \\
Total & 310 & 100 \\
\hline \hline
\end{tabular}

Tableau IV : Répartition des agents de santé selon l'état de connaissance des types les plus fréquents de cancers de l'enfant.

\begin{tabular}{lll}
\hline \hline $\begin{array}{l}\text { Quels sont les types les plus } \\
\text { fréquents de cancers de l'enfant ? }\end{array}$ & $\mathbf{n}$ \\
\hline \hline Leucémie et Lymphome & 57 & 18,39 \\
Cancer du sein & 9 & 2,90 \\
Neuroblastome & 2 & 0,64 \\
Rétinoblastome & 7 & 2,26 \\
Néphroblastome & 7 & 2,26 \\
Cancers osseux & 13 & 4,19 \\
Tumeurs cérébrales & 12 & 3,87 \\
Hépatoblastome & 00 & 00 \\
Rhabdomyosarcome & 00 & 00 \\
Cancers ORL & 2 & 0,64 \\
Ne sait pas & 201 & 64,84 \\
Total & 310 & 100 \\
\hline \hline
\end{tabular}

Tableau V : Répartition des agents de santé selon l'état de connaissance des différentes modalités de traitement des cancers de l'enfant sont

\begin{tabular}{llll}
\hline \hline $\begin{array}{l}\text { Les différentes modalités } \\
\text { traitement des } \\
\text { l'enfant }\end{array}$ & $\begin{array}{l}\text { cancers } \\
\text { de }\end{array}$ & & \\
\hline \hline Chirurgie & 16 & 5,16 \\
Radiothérapie & 11 & 3,55 \\
Chimiothérapie & 39 & 12,58 \\
Antibiothérapie & 8 & 2,58 \\
Phytothérapie & 2 & 0,65 \\
Pas de Traitement & 14 & 4,52 \\
Ne sait pas & 220 & 70,97 \\
Total & 310 & 100 \\
\hline \hline
\end{tabular}




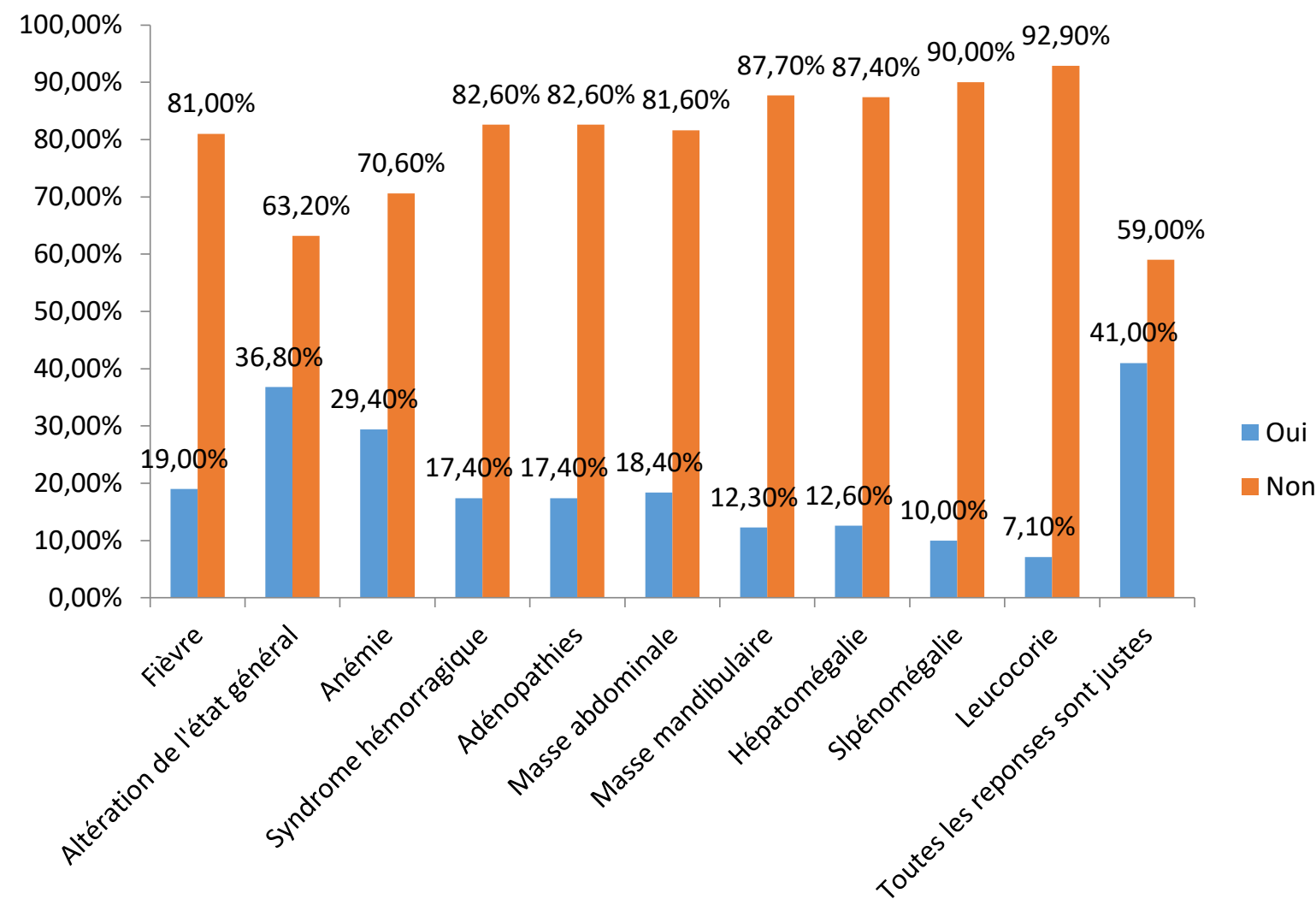

Figure 1 : Répartition des agents de santé selon la connaissance des manifestations cliniques des cancers de l'enfant.

Tableau Vl : Grade professionnel et état de connaissance sur la possibilité de guérison des cancers de l'enfant

\begin{tabular}{llll}
\hline & \multicolumn{3}{c}{ les cancers de l'enfant sont-ils curables : } \\
\cline { 2 - 4 } Grade Professionnel & Oui & Non & TOTAL \\
\hline \hline Aides-soignants & 16 & 17 & 33 \\
Assistant médicaux & 18 & 14 & 32 \\
Infirmier 1er cycle & 43 & 44 & 87 \\
Infirmier d'Etat & 26 & 16 & 42 \\
Matrones & 3 & 8 & 11 \\
Médecin généraliste & 24 & 8 & 32 \\
Médecin spécialiste & 22 & 9 & 31 \\
Sage-femme & 13 & 16 & 29 \\
Technicien de Laboratoire & 7 & 5 & 12 \\
TOTAL & 172 & 137 & 309 \\
\hline \hline
\end{tabular}

$p: 0,0497$ 